\title{
A novel scoring function for discriminating hyperthermophilic and mesophilic proteins with application to predicting relative thermostability of protein mutants
}

\author{
Yunqi Li ${ }^{1}$, C Russell Middaugh², Jianwen Fang ${ }^{1 *}$
}

\begin{abstract}
Background: The ability to design thermostable proteins is theoretically important and practically useful. Robust and accurate algorithms, however, remain elusive. One critical problem is the lack of reliable methods to estimate the relative thermostability of possible mutants.

Results: We report a novel scoring function for discriminating hyperthermophilic and mesophilic proteins with application to predicting the relative thermostability of protein mutants. The scoring function was developed based on an elaborate analysis of a set of features calculated or predicted from 540 pairs of hyperthermophilic and mesophilic protein ortholog sequences. It was constructed by a linear combination of ten important features identified by a feature ranking procedure based on the random forest classification algorithm. The weights of these features in the scoring function were fitted by a hill-climbing algorithm. This scoring function has shown an excellent ability to discriminate hyperthermophilic from mesophilic sequences. The prediction accuracies reached 98.9\% and $97.3 \%$ in discriminating orthologous pairs in training and the holdout testing datasets, respectively. Moreover, the scoring function can distinguish non-homologous sequences with an accuracy of $88.4 \%$. Additional blind tests using two datasets of experimentally investigated mutations demonstrated that the scoring function can be used to predict the relative thermostability of proteins and their mutants at very high accuracies ( $92.9 \%$ and 94.4\%). We also developed an amino acid substitution preference matrix between mesophilic and hyperthermophilic proteins, which may be useful in designing more thermostable proteins.

Conclusions: We have presented a novel scoring function which can distinguish not only HP/MP ortholog pairs, but also non-homologous pairs at high accuracies. Most importantly, it can be used to accurately predict the relative stability of proteins and their mutants, as demonstrated in two blind tests. In addition, the residue substitution preference matrix assembled in this study may reflect the thermal adaptation induced substitution biases. A web server implementing the scoring function and the dataset used in this study are freely available at http://www.abl.ku.edu/thermorank/.
\end{abstract}

\footnotetext{
* Correspondence: jwfang@ku.edu

${ }^{1}$ Applied Bioinformatics Laboratory, the University of Kansas, Lawrence, KS 66047, USA
}

\section{) Biomed Central}

(c) $2010 \mathrm{Li}$ et al; licensee BioMed Central Ltd. This is an Open Access article distributed under the terms of the Creative Commons Attribution License (http://creativecommons.org/licenses/by/2.0), which permits unrestricted use, distribution, and reproduction in any medium, provided the original work is properly cited. 


\section{Background}

Developing thermostable proteins has been a main focus of protein engineering because of its theoretical and practical significance [1-4]. Recently, computational protein design methods have been attracted much attention due to their potential cost and time savings over conventional directed evolution approaches $[3,5,6]$. These types of approaches utilize information extracted from protein sequences and/or 3D structures to predict favorable mutations that may enhance protein thermostability. Clearly, a key step in such approaches is the development of reliable methods for estimating the relative stability of possible mutants to identify favorable mutations. Such methods may also help better understand the proteinfolding problem since the ultimate outcome of protein folding is a native structure with the lowest free energy among many possible structures of a protein.

A common approach to study the thermostability of proteins is to perform comparative studies of the sequences and/or structures of (hyper)thermophilic proteins (HPs) and their mesophilic counterparts (MPs) [7-15] because there exists a direct positive correlation between the optimal growth temperature (OGT) of an organism and the melting temperature of its proteins, a key metric of protein thermostability [16,17]. Numerous studies have focused on amino acid composition changes caused by thermal adaptation at the whole genome level $[7,14,18]$. For example, Zeldovich et al. discovered that the total concentration of seven amino acids (INYWREL) in the proteins of an organism has a strong correlation with its OGT [14]. Overall, the proteins of thermophiles contain more charged and hydrophobic amino acid residues at the expense of polar ones $[7,14,18]$. The observed composition differences have prompted the development of predictive models discriminating HPs and MPs [19-21]. For example, Gromiha and Suresh applied 12 different classification algorithms and the best accuracy achieved reached 89\% [21].

Several amino acid substitution preference matrices have been created based on the sequence alignments of thermophilic proteins and their mesophilic homologues [22-24]. Analyzing these matrices and comparing sequences and structures of HPs and MPs have revealed a number of substitution trends potentially affecting thermostability $[7,8]$. Notable features include: an increased level of charged residues in hyperthermophilic proteins at the cost of polar residues on surface compared to their mesophilic homologs [23,25,26]; elevated levels of proline or $\beta$-branched amino acids in loops to reduce the freedom of coil regions [1,27]; a reduced number of residues in coil regions but increases in helix runs [28,29]; increased numbers of the high helix-propensity residues such as Lys and Glu, etc. [30]; an increased compactness of hydrophobic cores resulted in enhanced apolar interactions and interior packing [30-32]; and reduced deamidation probability by replacing Gln with Glu and Asn with Asp [33,34].

The goal of this study was to develop a scoring function for predicting relative thermostability of protein and their mutants using an integrated statistical and machine learning approach. We used HP/MP orthologs as research subjects because they are equivalent to mutants with multiple substitutions and, as discussed above, the difference between them may encode thermal-adaptation mechanisms. Thus a scoring function which can distinguish HP/MP orthologs is presumably able to rank the relative stability of a protein and its mutants, a key step for designing more thermostable proteins.

In this study, we first constructed a set of 540 nonredundant hyperthermophilic-mesophilic protein ortholog pairs. Since our dataset is significantly bigger than previous studies, we then calculated a substitution preference matrix using an established approach $[11,12,22-24]$. We used a feature selection procedure based on the random forest algorithm to identify sequence-based features important to pairwise discrimination of hyperthermophilic and mesophilic protein orthologs. We then used a hill-climbing algorithm to fit a scoring function based on a linear combination of these important discriminating features. Finally, we applied the scoring function to two experimental datasets to demonstrate that this scoring function can indeed be used to rank thermostability of protein mutants with high accuracy.

\section{Methods}

\section{Datasets}

We downloaded all protein sequences of nine organisms, including four hyperthermophilic and five mesophilic organisms (Table 1) from the NCBI http://www. ncbi.nlm.nih.gov/. To identify HP-MP ortholog pairs, we

Table 1 The list of organisms whose proteins were used to generate the non-redundant hyperthermophilic (upper) and mesophilic (bottom) orthologous pairs (adopted from [48]).

\begin{tabular}{lll}
\hline Organism & Number of proteins & OGT $\left({ }^{\circ} \mathbf{C}\right)$ \\
\hline Aquifex aeolicus VF5 & 1560 & 96 \\
Methanocaldococcus jannaschii DSM & 1786 & 85 \\
Thermotoga maritima MSB8 & 1858 & 80 \\
Pyrococcus abyssi GE5 & 1898 & 103 \\
\hline Corynebacterium glutamicus ATCC & 2993 & $30-40$ \\
Escherichia coli K12 & 4237 & 37 \\
Mycobacterium tuberculosis H37RV & 3991 & 37 \\
Bacillus halodurans C-125 & 4066 & $25-35$ \\
Streptococcus pneumoniae TIGR4 & 2094 & $30-35$ \\
\hline
\end{tabular}


performed BLAST searches for all MP sequences against all HP sequences [35]. The following conservative criteria were used to identify putative orthologs:

- Reciprocal best BLAST hits with the e-values in BLAST searches less than $10^{-10}$;

- The difference in the number of residues is less than $5 \%$ of the shorter sequence so that only small insertions/deletions were allowed;

- Higher than $30 \%$ amino acid sequence identity.

In addition, we removed transmembrane proteins, predicted by TMHMM $2.0 \mathrm{http} / / / \mathrm{www} . \mathrm{cbs}$.dtu.dk/services/TMHMM/, because they often use different strategies from soluble proteins to survive under high temperature environments [36]. Furthermore, to reduce the statistical bias caused by redundancy, we clustered paralogues using the blastclust program available in the BLAST package [35]. The minimum length coverage of blastclust was set to 0.5 and the sequence similarity threshold was set to 0.25 . Sequences longer than 600 or shorter than 50 residues were also removed. The final dataset consists of 540 non-redundant HP-MP ortholog pairs. Pfam http:// pfam.sanger.ac.uk/ domain scans of these proteins confirmed, as expected, that the two proteins of each ortholog pair contain the same domains. Thus the selected pairs are very likely true orthologs.

We also used a set of 373 structurally well-aligned protein pairs from (hyper)thermophilic and mesophilic organisms compiled by Glyakina et al. for testing purpose [37]. The dataset includes 63 hyperthermophilic and 310 thermophilic proteins.

\section{Amino acid substitution matrix}

The amino acid residue substitution matrix was constructed following an established procedure [11,12,22-24]. In brief, we counted each of the 380 types of amino acid residue substitutions in the BLAST sequence alignments of all MP/HP pairs. Substitutions in converting MPs to HPs are considered as the "forward" direction. Two-tail binomial statistics were used to estimate the statistical significance of the asymmetry of the forward and reverse substitutions of any given pair of amino acids [23].

\section{Two sets of experimentally investigated protein mutations}

In addition to the protein pairs mentioned before, we used two independent datasets for additional testing. The first set contains two wild-type adenylate kinases (ADKs) from Methanococcus Voltae and Methanococcus Jannaschii, and a series of chimeric proteins generated from these two enzymes [38]. These proteins share significant sequence identity but differ in their thermostability (Table 2). The second dataset was collected by Montanucci and colleagues [20]. It contains 10 wild type proteins and 14 mutants manifesting thermal stability changes (Table 3 ). All protein sequences in these two tests were subjected to BLAST searches against all sequences in the 540 ortholog pairs. Only one protein, BsCSP (GI: 16077975), showed greater than $25 \%$ similarity to one of the protein sequences in the

Table 2 Two wild-type ADKs and a series of chimeric enzymes generated from these two enzymes[38].

\begin{tabular}{lllll}
\hline Seq_ID & Comm_meso & Comm_hyp & $\mathbf{T}_{\mathbf{m}}\left({ }^{\circ} \mathbf{C}\right)$ & Ranking \\
\hline MJA & 0 & 62 & 103 & 8 \\
V36J & 9 & 53 & 98 & 7 \\
J160V & 9 & 53 & 96 & 6 \\
JVJ & 37 & 25 & 89 & 4 \\
VJV & 20 & 42 & 82.5 & 5 \\
V160J & 51 & 11 & 74 & 2 \\
J36V & 53 & 9 & 73 & 3 \\
MVO & 62 & 0 & 69 & 1 \\
\hline
\end{tabular}

Comm_meso and comm_hyp are the counts of the identical residues in the MP sequence MVO and the HP sequence MJA, respectively. The last column is the relative stability ranked by our scoring function (from least to most stable).

Table 3 The ranking of relative thermostability of wild type proteins and their mutated sequences using the scoring function.

\begin{tabular}{|c|c|c|c|}
\hline Protein name & length & $\mathrm{T}_{\mathrm{m}}\left({ }^{\circ} \mathrm{C}\right)$ & Ranking \\
\hline Dmeh (Gl: 640374) & 51 & 49 & 1 \\
\hline Dmeh_UMC & 51 & 99 & 2 \\
\hline Dmeh_UVF & 51 & 99 & 3 \\
\hline BsCSP (Gl: 16077975) & 67 & 53.8 & 1 \\
\hline BsCSP_mt1 & 67 & 69.7 & 2 \\
\hline BsCSP_mt2 & 67 & 83.7 & 3 \\
\hline PhyA (Gl: 464382) & 467 & 55 & 1 \\
\hline PhyA_mt18 & 467 & 62 & 2 \\
\hline PhyA_mt24 & 467 & $62+$ & 3 \\
\hline PTDH (Gl: 194552172) & 336 & 39 & 1 \\
\hline PTDH_12x & 336 & 59.7 & 2 \\
\hline PTDH_opt14 & 336 & 64.4 & 3 \\
\hline CbADH (Gl: 187935035) & 351 & 64.5 & 1 \\
\hline CbADH_Q100P & 351 & 76 & 2 \\
\hline$\overline{\beta-G U S ~(G I: ~ 868020) ~}$ & 602 & 45 & 1 \\
\hline$\beta$-GUS_TR3337 & 602 & 65 & 2 \\
\hline FAOX (GI: 20302586) & 372 & 37 & 1 \\
\hline FAOX_TE & 372 & 45 & 2 \\
\hline Shble (Gl: 3891709) & 121 & 67.4 & 1 \\
\hline Shble_HTS & 121 & 85.1 & 2 \\
\hline ECHPH (GI: 12539) & 341 & 51 & 1 \\
\hline EcHPH_hph5 & 341 & 67 & 2 \\
\hline PDAO (Gl: 129305) & 347 & 45 & 2 \\
\hline PDAO_F42C & 347 & 55 & 1 \\
\hline
\end{tabular}

The data were originally collected by Montanucci et al. [20]. The sequence of $\mathrm{cbADH}$ was retrieved from the original literature by Goihberg et al.[49]. 
540 pairs. Thus, these proteins can be used as independent testing datasets.

\section{Features}

A set of 83 features derived from protein sequences was calculated using various software programs or in-house scripts (Table 4, more information about these feature is available in the additional file 1). These features can be roughly classified into two groups. The features in the first group, denoted as $c_{k}$, are the absolute counts of amino acid residues or other properties. The features in the second group, labeled as $x_{k}$, are the chain length normalized values of the features in the first group. Although including structure-based features may be of great help in understanding the mechanisms of mutagenesis induced protein stabilization, the vast majority of proteins lack solved structures. Therefore we only investigated the contributions from sequence-based features in this work. Furthermore, the theory that the sequence of a protein determines its structure suggests that the knowledge extracted from the sequence may be sufficient to distinguish proteins with different thermostability. Besides the general information extracted from the sequence, we also included several predicted features which were obtained by mature and widely-used algorithms, such as those used to predict secondary structure [39] and exposed/buried residues [40] (Table 4).

\section{Random Forest}

The random forest algorithm is an ensemble technique that utilizes the results of hundreds or even thousands of decision trees to perform classification or regression
[41]. Each of the member trees is built on a bootstrap sample from the training data and utilizes a random subset of available variables. The algorithm has been applied in broad classification tasks and has frequently demonstrated superior performance compared to other classification algorithms [42,43]. It is robust and particularly suitable for classifying high-dimensional and noisy data. One very useful feature of the algorithm is that it offers several methods to assess the importance of various features based on their contributions to the correctness of the resulting classification [41]. In this study, we used the Gini importance to rank the importance of all used features. The Gini importance is the summation of the Gini impurity decreases in node splits made on the feature over all trees in the model. The Gini impurity is a common metric to measure the degree of impurity [44]. It is defined as:

$$
I(A)=1-\sum_{k=1}^{m} p_{k}^{2}
$$

where $k=(1,2, \ldots, \mathrm{m})$ are possible classes and $p_{k}$ is the relative frequency of class $k$ in a node $\mathrm{A}$. Therefore $I(\mathrm{~A})$ equals to zero when all cases in the node belong to a single class and reaches its maximum when cases are equally distributed to all classes.

We used a random forest package implemented in the $\mathrm{R}$ environment for this study http://cran.r-project.org/web/ packages/randomForest/index.html. Random forest models are usually insensitive to the model parameters [41]. Consequently the default parameters were used in the study.

Table 4 The list of the 83 features used in the study.

\begin{tabular}{|c|c|c|}
\hline Protein feature & $\begin{array}{l}\text { Number of } \\
\text { Features }\end{array}$ & Source \\
\hline Sequence length $(L)$ & 1 & In-house script \\
\hline Count and composition of amino acids & 40 & In-house script \\
\hline Number and percentage of positive, negative and all charged residues, as well as the net charges & 8 & In-house script \\
\hline $\begin{array}{l}\text { Number and percentage of small ( } T \text { and } D) \text {, tiny }(G, A, S \text { and } P) \text {, aromatic }(F, H, Y, W) \text {, aliphatic, hydrophobic } \\
\text { and polar residues }\end{array}$ & 12 & In-house script \\
\hline Number and percentage of residues which can form hydrogen bond in sidechain & 2 & In-house script \\
\hline Number of sulfide atoms & 1 & In-house script \\
\hline Average solubility of amino acids in aqueous solutions under room temperature & 1 & ** \\
\hline The average of the maximum solvent accessible surface area (ASA) of each amino acid & 1 & Eisenhaber[50] \\
\hline Predicted isoelectric point (pl) of the protein, the average pl on all residues (pla) & 2 & ProtParam[51] \\
\hline Instability index and instability class & 2 & \\
\hline Aliphatic index & 1 & \\
\hline Gravy hydropathy index & 1 & \\
\hline Composition of the predicted secondary structure residues & 3 & Psipred[52] \\
\hline Predicted percentages of buried/exposed residues & 2 & Accpro[40] \\
\hline The overall length and percentage of all coils, rem465, and hotloop & 6 & disEMBL[53] \\
\hline
\end{tabular}

**Obtained from The Merck Index, Merck \& Co., Inc., Whitehouse Station, NJ 12 (1996). 


\section{Results and Discussion}

In this section, we first report a MP/HP residue substitution preference matrix generated from the BLAST pairwise alignments of MP and HP orthologs. Feature selection using the random forest algorithm is then described, followed by the scoring function construction. The performance of the scoring function in discriminating hyperthermophilic and mesophilic proteins was estimated with a set of holdout testing dataset. Finally, the application of the scoring function in predicting relative stability of proteins and their mutants is presented.

\section{Amino acid composition}

The overall differences in amino acid composition between HPs and MPs are consistent with previous reports (Table 5) [7,11,22-24,45]. Based on the p-values from unpaired and paired $\mathrm{t}$-test, the most significantly increased residues in HPs include Lys, Glu, Tyr, and Ile, while reduced residues include Gln, His, Ala, and Thr.

The 540 HPs consist of 426 bacteria and 114 archaea proteins while all MPs are from bacteria. In order to rule out the possibility that the different domains cause bias toward residue composition and the final results, we calculated the correlation coefficients of the amino acid compositions between HPs from archaea and MPs, HPs from bacteria and MPs, and HPs from bacteria and HPs from archaea. The $\mathrm{R}$ values of the correlations are $0.779,0.828$, and 0.968 , respectively (Figure 1).
Therefore, the composition difference possibly attributed to bacteria $v s$. archaea domains isn't as significant as the contributions by thermal adaptation.

\section{Amino acid residue substitutions}

All 380 residue substitutions are reported in Figure 2. We also calculated the ratio of each substitution to the opposite replacement. Substitutions with statistically significant bias $\left(\mathrm{p}<10^{-10}\right)$ are shown in bold. Red cells are substitutions favored in the MP to HP direction while blues are favored in the opposite direction. There are 84 (22\%) significantly biased substitutions and 44 of them are in the direction from MP to HP. The overall trends of the substitution preferences are consistent with previous studies [22-24]. For example, charged residues, especially Lys and Glu, gain significantly in HPs at the cost of uncharged polar residues such as Ser, Gln, and Tyr.

Many of the significant substitution asymmetries are consistent with various proposed protein thermo stability mechanisms. For example, Asp is preferred to be substituted by Glu or Lys in the direction from MP to HP, both are helix favored while Asp is coil favored. This is consistent with previous findings that in general HPs contain more helical regions at the cost of disordered regions than MPs $[28,29]$. There is a strong preference for Ser, Thr, Asn and Gln to be substituted by Lys and Glu in HPs, which can be explained by the observed significant reduction of polar non-charged

Table 5 Comparison of the composition of the amino acids in hyperthermophilic and mesophilic proteins and their significance $p$-values of $t$-test and paired $t$-test.

\begin{tabular}{|c|c|c|c|c|}
\hline Amino acid & Composition in HP & Composition in MP & p-value (t-test) & $p$-value (paired $t$-test) \\
\hline $\mathrm{S}$ & $0.044 \pm 0.016$ & $0.050 \pm 0.015$ & $9.60 \times 10^{-9}$ & $5.61 \times 10^{-12}$ \\
\hline $\mathrm{Q}$ & $0.019 \pm 0.011$ & $0.037 \pm 0.015$ & $6.81 \times 10^{-85}$ & $1.24 \times 10^{-94}$ \\
\hline $\mathrm{N}$ & $0.035 \pm 0.014$ & $0.035 \pm 0.015$ & 0.88 & 0.85 \\
\hline$\overline{\mathrm{T}}$ & $0.042 \pm 0.014$ & $0.055 \pm 0.016$ & $1.02 \times 10^{-40}$ & $2.44 \times 10^{-56}$ \\
\hline $\bar{C}$ & $0.009 \pm 0.011$ & $0.010 \pm 0.011$ & 0.36 & 0.08 \\
\hline $\bar{G}$ & $0.075 \pm 0.019$ & $0.079 \pm 0.020$ & $9.14 \times 10^{-4}$ & $9.68 \times 10^{-10}$ \\
\hline $\bar{A}$ & $0.066 \pm 0.023$ & $0.080 \pm 0.028$ & $3.41 \times 10^{-48}$ & $1.08 \times 10^{-87}$ \\
\hline$\overline{\mathrm{H}}$ & $0.017 \pm 0.010$ & $0.024 \pm 0.013$ & $3.00 \times 10^{-20}$ & $4.64 \times 10^{-40}$ \\
\hline $\bar{M}$ & $0.024 \pm 0.011$ & $0.026 \pm 0.010$ & 0.02 & $3.00 \times 10^{-3}$ \\
\hline $\bar{Y}$ & $0.033 \pm 0.014$ & $0.027 \pm 0.013$ & $6.10 \times 10^{-15}$ & $4.47 \times 10^{-31}$ \\
\hline $\bar{F}$ & $0.038 \pm 0.015$ & $0.033 \pm 0.014$ & $3.00 \times 10^{-8}$ & $1.29 \times 10^{-14}$ \\
\hline $\mathrm{V}$ & $0.086 \pm 0.021$ & $0.082 \pm 0.020$ & $2.32 \times 10^{-4}$ & $5.36 \times 10^{-7}$ \\
\hline $\bar{L}$ & $0.089 \pm 0.021$ & $0.089 \pm 0.022$ & 0.73 & 0.59 \\
\hline$P$ & $0.041 \pm 0.015$ & $0.040 \pm 0.014$ & 0.39 & 0.16 \\
\hline I & $0.077 \pm 0.020$ & $0.066 \pm 0.019$ & $6.46 \times 10^{-20}$ & $3.15 \times 10^{-29}$ \\
\hline $\bar{W}$ & $0.008 \pm 0.007$ & $0.007 \pm 0.007$ & 0.05 & $3.00 \times 10^{-3}$ \\
\hline $\bar{D}$ & $0.050 \pm 0.015$ & $0.057 \pm 0.016$ & $1.92 \times 10^{-12}$ & $5.64 \times 10^{-22}$ \\
\hline $\bar{E}$ & $0.097 \pm 0.023$ & $0.079 \pm 0.022$ & $6.73 \times 10^{-38}$ & $5.63 \times 10^{-75}$ \\
\hline $\bar{K}$ & $0.091 \pm 0.023$ & $0.060 \pm 0.023$ & $1.21 \times 10^{-87}$ & $5.25 \times 10^{-117}$ \\
\hline $\mathrm{R}$ & $0.056 \pm 0.023$ & $0.055 \pm 0.023$ & 0.57 & 0.36 \\
\hline
\end{tabular}




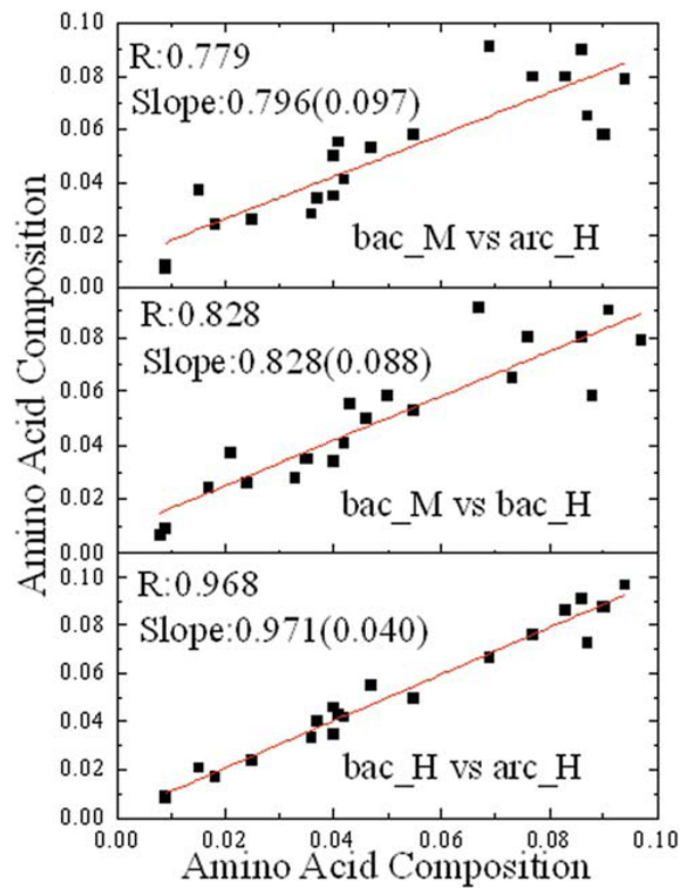

Figure 1 The pariwise comparisons of amino acid compositions in the three different sets of proteins. The solid lines show the best-fit of linear regression lines with regression coefficient and slope displayed and the dash lines show the orthogonal line. Bac_M, arc_H and bac_H are proteins from mesophilic bacteria, hyperthermophilic archaea and bacteria, respectively.

residues $[23,26]$ and deamidation vulnerable residues $[33,34]$ in HPs. Leu is preferred to be substituted by Ile to enhance thermo stability. This is consistent with the finding that increasing $\beta$-branched amino acids in loop regions enhance protein thermostability $[1,27]$.

It is worth mentioning that the significance threshold $\left(\mathrm{p}<10^{-10}\right.$, Fisher's exact test [46]) used in this study was significantly more stringent than the criteria used in previous studies (e.g. $\mathrm{p}<10^{-2}$ ) because we used approximately five times as many HP/MP pairs as previous studies. The ratios of forward-to-reverse changes for these substitutions were also calculated based on more examples than in previous studies. For example, the matrix reported by Haney et al. contained 72 residue replacements with no or only single instances [23]. In our matrix, the minimum number is 3 and there are only 14 substitutions with less than 10 examples. Therefore the ratios in this matrix may better reflect thermal adaptation induced substitution biases and should be useful in designing thermostable proteins.

\section{Ranking features using a random forest algorithm}

The analysis of the residue substitution preference between MPs and HPs clearly indicates that different residues contribute to protein thermostability differentially. In this section, we describe a procedure for ranking the importance of all 83 features derived from protein sequences in discriminating MPs and HPs using the random forest algorithm.

A standard five-fold cross validation procedure was used to determine the importance of features and develop the scoring function. We randomly split the 540 pairs into five equal portions. We used four portions as training datasets and reserved the remaining portion for testing purposes. We then constructed a random forest model with 3000 trees for discriminating these 432 ortholog protein pairs in the training set and then used the Gini importance to rank these features. The procedure was repeated four more times and each time a different portion was used as the testing dataset. We found that the results from all five runs were very consistent. All features were ranked by their average importance and top 25 are shown in Figure 3. The levels of glutamine and lysine are most important among the 83 features used in this study, followed by the percentage of positively charged residues. We also noticed that the features normalized by sequence length are consistently more important than the corresponding absolute counts. Thus, we only used the normalized features in the scoring function. Interestingly, all predicted features, such as secondary structure, the ratio of exposed to buried residues, and the disordered region predictions, failed to appear in the 25 most important features.

\section{Developing the scoring function}

We first calculated the relative feature difference $\Delta x_{\mathrm{i}}$, which is defined as:

$$
\Delta x_{i}=\frac{x_{i}(\mathrm{seq} 1)-x_{i}(\mathrm{seq} 2)}{x_{i}(\mathrm{seq} 1)+x_{i}(\mathrm{seq} 2)}
$$

where $x_{i}\left(\right.$ seq1) and $x_{i}$ (seq2) are the values of the $i$ th feature from the first sequence and the second sequence, respectively. We plotted the cumulative curves of the relative feature difference $\Delta x_{\mathrm{i}}$ of the ten most significant normalized features in the training dataset (Figure 4). In this plot, all cumulative curves show typical sigmoid shapes in which the inflexion points are located in the curve at the half height, i.e., the cumulative counts are equal to half of the total counts.

We constructed the scoring function by a linear combination of the ten most important features. The scoring function can be written as:

$$
\text { Score }(\operatorname{seq} 1, \operatorname{seq} 2)=\sum_{i} w_{i} \Delta x_{i}
$$

where $i$ runs over all 10 features are used in the scoring function and $w_{i}$ is the weight for each feature. The 


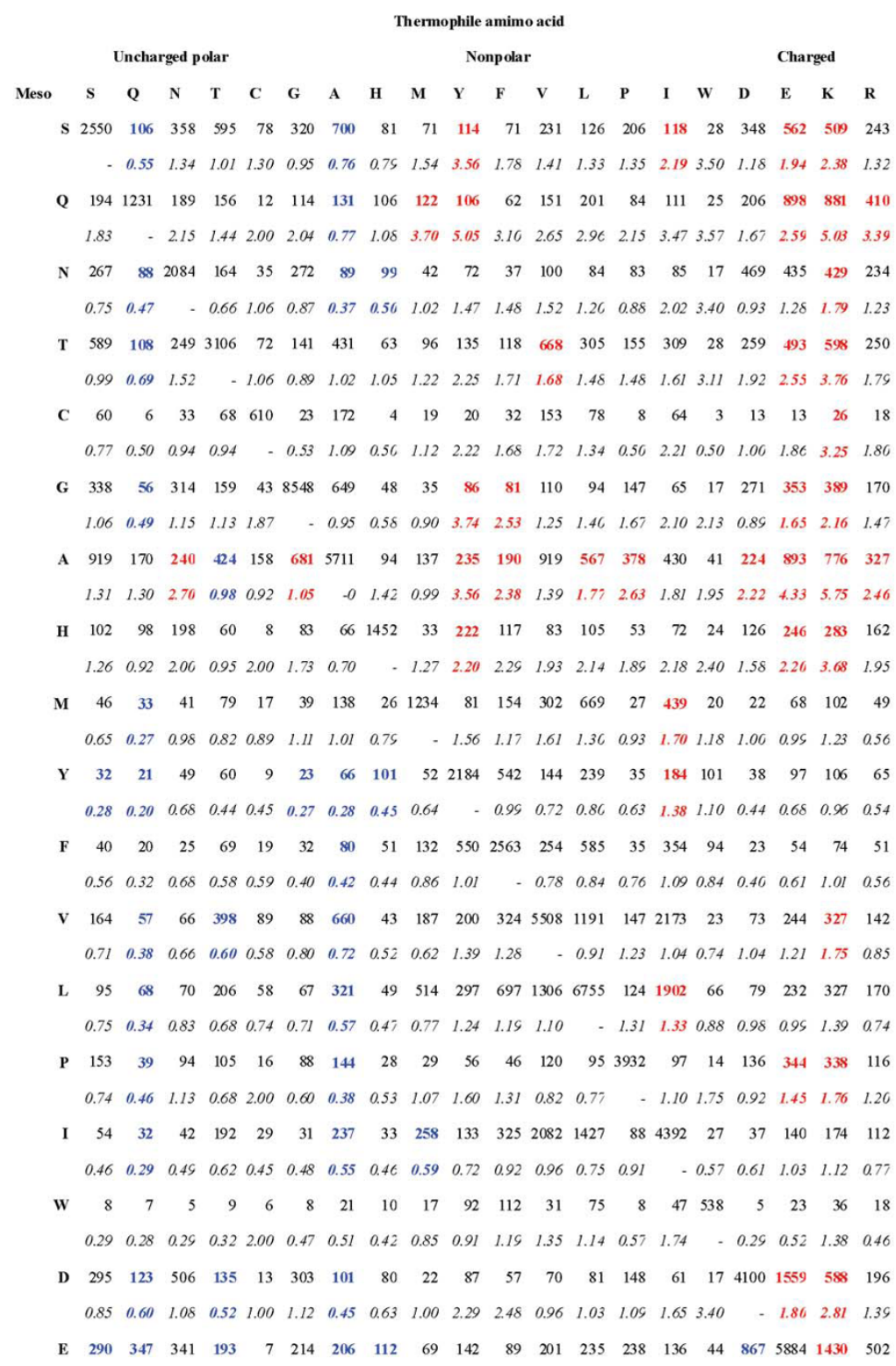

Figure 2 Amino acid substitutions between mesophilic and hyperthermophilic proteins. The top number in each cell is the observed substitution instances and the bottom one (in italics) is the ratio of the number of the substitution cases to the opposite substitution. Significant biased substitutions ( $p$-value $<10^{-10}$, two-sided Fisher's exact test) are highlighted in bold. Red cells are significant HP favored substitutions while blues are MP favored.

sign of the weight of each feature was determined by the location of the inflexion point of its cumulative curve: positive for features located to the left and negative for those to the right of the zero-difference line. Thus the signs of $x_{-} \mathrm{K}, x_{-} \mathrm{E}, x_{-}$pos, $x_{-}$charge, and ASA are positive, and negative for $x_{-}$small, $x_{-}$tiny, $x_{-} \mathrm{A}, x_{-} \mathrm{Q}$ and $x_{-} \mathrm{T}$. We then used a hill-climbing algorithm to fit the weights of these features. The absolute values of all weights were restricted to the range of 0 to 1 . We randomly assigned an initial weight to each feature and counted the number of correctly ranked ortholog pairs.
The weights were then randomly updated and the number of correct ranks was recounted. The new weights were kept if they resulted in more correctly ranked ortholog pairs; otherwise the weights were rolled back to the previous values. This procedure was repeated $5 \times$ $10^{7}$ times and the batch of weights which maximized the number of positive score values was recorded. To check whether the optimization procedure was trapped in a local maximum, we repeated the procedure four more times using different random seeds. The results were very similar and thus we simply used the average 


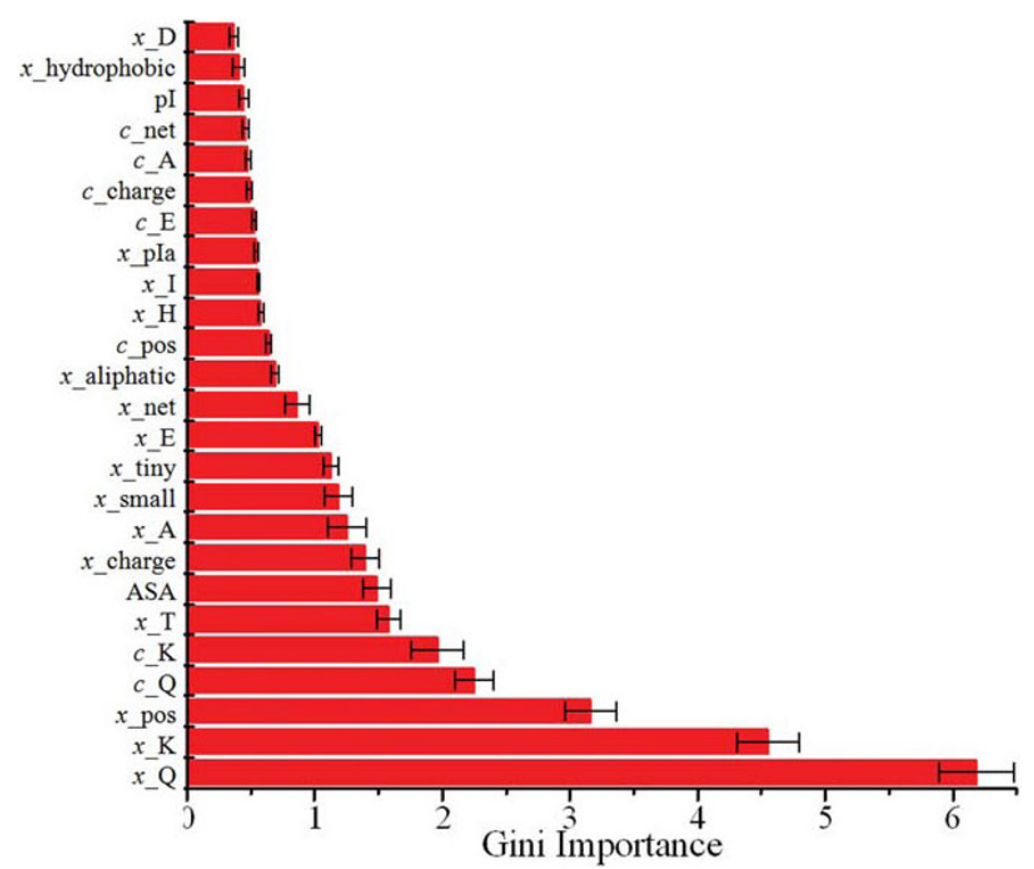

Figure 3 The $\mathbf{2 5}$ most important features ranked by the Gini importance of the random forest algorithm. The prefixes $C_{-}$and $x_{-}$of each feature indicate that the feature is an absolute count or normalized value, respectively.

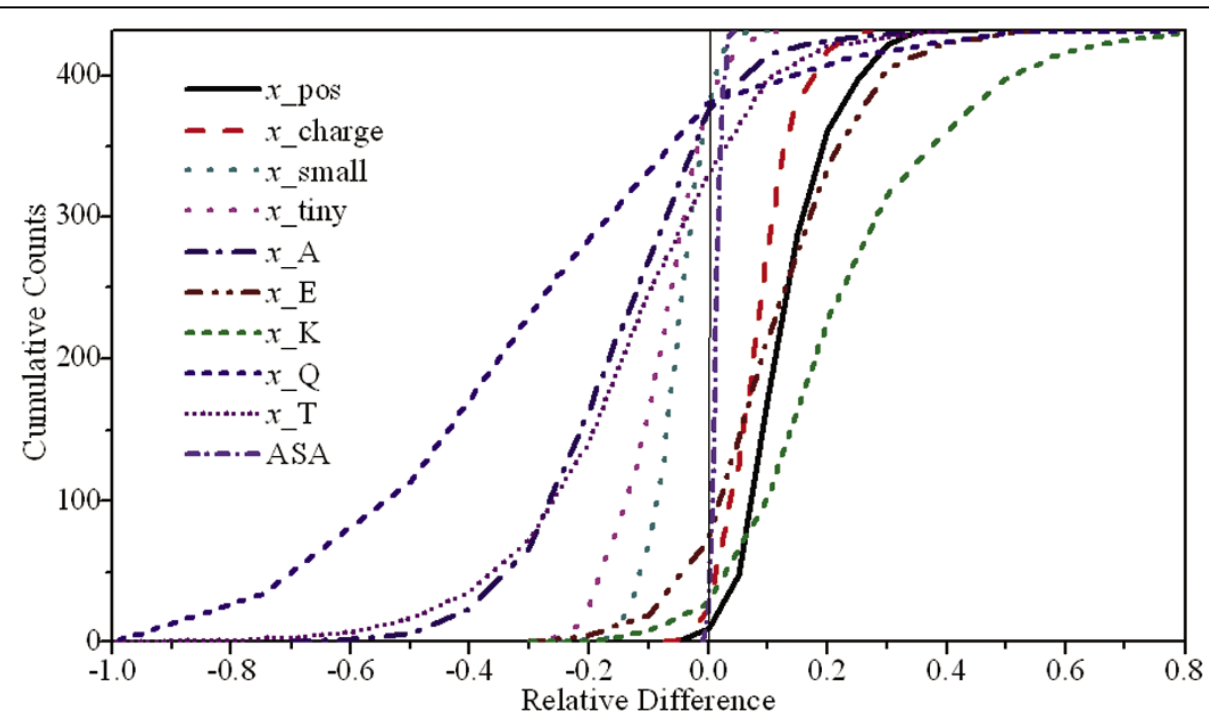

Figure 4 The cumulative curves of the 10 most important features against the relative difference between hyperthermophilic and mesophilic sequences.

of the weights in the scoring function. We then used the same procedure to develop four more scoring functions, each for one of remaining training datasets.

\section{The discrimination ability of the scoring function}

We calculated the accuracies of the discriminations made by the five scoring functions on their corresponding training datasets. The scoring functions using optimized weights were able to distinguish in average $427.1 \pm 1.9$ out of 432 (98.9\% accuracy) ortholog protein pairs in the training datasets. We then tested each of the scoring functions with its corresponding holdout testing dataset. Out of 108 protein pairs in the testing sets, on average $105.1 \pm 0.5$ pairs were correctly ranked $(97.3 \%$ accuracy). This was very close to the accuracy obtained from the training sets $(98.9 \%)$. Thus the scoring function 
Table 6 The final weights of the ten features used in the scoring function.

\begin{tabular}{|c|c|c|c|c|c|c|c|c|c|c|}
\hline Feature & $x \_\mathrm{K}$ & $x_{-} \mathrm{E}$ & x_pos & x_charge & ASA & x_small & $x$ _tiny & X_A & X_Q & $x_{-} T$ \\
\hline Weight & 0.75 & 0.20 & 0.80 & 0.20 & 0.90 & -0.20 & -0.20 & -0.30 & -0.10 & -0.20 \\
\hline
\end{tabular}

is robust and able to discriminate a broad spectrum of HP and MP homologous protein pairs.

The average weights for each feature determined in all five training procedures are quite consistent. Thus we simply use the averages of these weights in the final scoring function (Table 6). It is noteworthy that while the signs of the weights indicate whether the features are favorable or not in hyperthermophilic proteins, their absolute values are not significant since the features are not normalized to a common scale.

We also applied the scoring function to discriminating (hyper)thermophilic and mesophilic proteins in the Glyakina dataset [37]. Our scoring function was able to correctly discriminate not only $59 \mathrm{HP} / \mathrm{MP}$ pairs $(93.7 \%$ accuracy), but also 238 thermophilic and mesophilic pairs (76.8\% accuracy). The list of these proteins and their scores are provided in Table S2 in the additional file 1 . We believe that the difference of the accuracy between hyperthermophilic and thermophilic proteins was caused by the different stabilization mechanisms of hyperthermophilic and thermophilic proteins, as previously suggested in literature $[17,31]$.

\section{Discriminating non-homologous protein pairs}

Encouraged by the results in the above test, we further challenged the scoring function in discriminating nonhomologous HP/MP protein pairs. In this test, we compared each HP protein sequence against all MP sequences. The overall accuracy of these $540 * 540$ pairwise comparisons was $88.4 \%$. Such a high accuracy in discriminating non-homologous HP and MP sequences confirms that HP sequences share some common sequential patterns to generate sufficient stability at elevated temperature.

Application in ranking the thermostability of proteins and their mutants

The first test was carried out on two wild-type ADKs and a series of chimeric enzymes generated from these two enzymes [38]. The predicted ranking of thermo-stability using the scoring function is highly consistent with the experimental results (Table 2). In all $28\left(C_{8}^{2}\right)$ pairwise comparisons, only two resulted in incorrect predictions (92.9\% accuracy). Moreover, the two inaccurate predictions included one between VJV and JVJ in which the Tm differed by only $6.5^{\circ} \mathrm{C}$, and the other between V160J and J36V in which the Tm differed by just $1^{\circ} \mathrm{C}$, probably not an experimentally detectable difference.
In the second test, we used a batch of sequences collected by Montanucci, et al [20]. The sequence lengths, the GI numbers of the wild-type proteins, and their melting temperatures are listed in Table 3. We used the scoring function to rank the relative thermostability of wild-type proteins and their mutants. In the case of proteins with two mutants, the relative stability of these mutants was also predicted. Overall there were 18 pairwise comparisons between these wild proteins and their mutants. The scoring function achieved an accuracy of $94.4 \%(17 / 18)$. The wrong prediction was for protein PDAO and its mutant (Table 3 ). It is a single mutation (F42C) and the difference in $\mathrm{Tm}$ is moderate $\left(10^{\circ} \mathrm{C}\right)$.

Overall, the scoring function has consistently demonstrated a remarkable ability to rank the relative thermostability of proteins and their mutants. Thus a website http://www.abl.ku.edu/thermorank/ was created and made freely available to the general public.

\section{Comparison with other Methods}

The current study differs at the level of information granules from previous work focused amino acid composition differences between thermophilic and mesophilic organisms $[7,14,18]$. We focused on the differences between HP and MP ortholog pairs instead of on the differences between thermophilic or mesophilic proteins at the genome level. The difference between these two approaches is similar to the one between unpaired and paired two-sample $t$-tests. While previous studies have succeeded in revealing the overall changes caused by thermal adaptation at the genome level, our study has further focused on the protein level. Such an approach may reduce or eliminate the effects of confounding factors such as protein families because it is well established that the amino acid composition may vary in different protein classes [47]. In addition, a protein level study may be more relevant to designing stable proteins because orthologs are essentially mutants with multiple mutations.

To compare the performance of our algorithm to other approaches is difficult because very few algorithms have been developed to rank the relative thermostability of HP/MP orthologous pairs and these studies have used different datasets $[20,48]$. TargetStar, a scoring function based on the analysis of 1006 decoy structures for a given protein, can discriminate HP/MP orthologs pairs with $77 \%$ accuracy [48]. Recently, Montanucci and colleagues reported a SVM model which achieves $88 \%$ accuracy on a set of redundancy-reduced HP/MP pairs 
[20]. The SVM model used residue and dipeptide compositions as predictive features. Thus, the $97.3 \%$ predictive accuracy on the test dataset of our scoring function is considerably higher than the reported accuracies of both previous methods. Moreover, in the application of predicting the relative thermostability of proteins and their mutants, our approach achieved an accuracy of 94.4\% (17/18) in the second blind test set, which represents one more correct prediction than Montanucci et $a l$. on the same dataset [20].

\section{Conclusions}

We have presented a novel scoring function which can distinguish not only HP/MP ortholog pairs, but also non-homologous pairs at high accuracies. Most importantly, it can be used to accurately predict the relative stability of proteins and their mutants, as demonstrated in two blind tests. In addition, the residue substitution preference matrix assembled in this study may better reflect the thermal adaptation induced substitution biases than previous studies because a larger dataset was used. The large set of HP/MP is available in the supplementary website and should be useful to other researchers for further development of novel algorithms in this area.

\section{Additional file 1: Supplementary Table S1, S2, and Figure S1. This}

file contains the following contents: 1. Table S1. Detailed description of the 10 features used to construct the scoring function. 2. Table S2. A test of the discriminative ability for the scoring function on a dataset containing 63 hyperthermophilic-mesophilic protein pairs and 310 thermophilic-mesophilic protein pairs. 3. Figure S1. The ROC curve of the scoring function in discrimination of 540 pairs of ortholog protein sequences accumulated from the 5 -fold cross testing set.

Click here for file

[http://www.biomedcentral.com/content/supplementary/1471-2105-1162-S1.PDF]

\section{Abbreviations}

HP: hyperthermophilic protein; MP: mesophilic protein; OGT: optimal growth temperature; Tm: melting temperature.

\section{Acknowledgements}

We thank Drs. Robert Hanzlik and Yang Zhang of the University of Kansas for their valuable scientific input. We also wish to thank Dr. Montanucc Ludovica for her kindly sharing the test sequences.

Funding: This work was partially supported by NIH grants P01 AG12993 (PI: E. Michaelis).

\section{Author details}

${ }^{1}$ Applied Bioinformatics Laboratory, the University of Kansas, Lawrence, KS 66047, USA. ${ }^{2}$ Department of Pharmaceutical Chemistry, the University of Kansas, Lawrence, KS 66047, USA.

\section{Authors' contributions}

JWF conceived the project. YL and JWF carried out the study with input from CRM. YL, CRM and JWF drafted the manuscript. All authors have read and approved the final manuscript.
Received: 28 September 2009

Accepted: 28 January 2010 Published: 28 January 2010

\section{References}

1. Sterner R, Liebl W: Thermophilic adaptation of proteins. Critical Reviews in Biochemistry and Molecular Biology 2001, 36:39-106.

2. Dahiyat BI: In silico design for protein stabilization. Current Opinion in Biotechnology 1999, 10:387-390

3. Korkegian A, Black ME, Baker D, Stoddard BL: Computational thermostabilization of an enzyme. Science 2005, 308:857-860.

4. Lazar GA, Marshall SA, Plecs JJ, Mayo SL, Desjarlais JR: Designing proteins for therapeutic applications. Curr Opin Struct Biol 2003, 13:513-518.

5. Schweiker KL, Makhatadze GI: A Computational Approach for the Rational Design of Stable Proteins and Enzymes: Optimization of Surface ChargeCharge Interactions. Methods in Enzymology: Computer Methods 2009, 454(Pt A):175-211.

6. Liao J, Warmuth MK, Govindarajan S, Ness JE, Wang RP, Gustafsson C, Minshull J: Engineering proteinase $\mathrm{K}$ using machine learning and synthetic genes. Bmc Biotechnol 2007, 7:16.

7. Zhou XX, Wang YB, Pan YJ, Li WF: Differences in amino acids composition and coupling patterns between mesophilic and thermophilic proteins. Amino Acids 2008, 34:25-33.

8. Razvi A, Scholtz JM: Lessons in stability from thermophilic proteins. Protein Science 2006, 15:1569-1578.

9. Menendez-Arias L, Argos P: Engineering protein thermal stability. Sequence statistics point to residue substitutions in alpha-helices. J Mol Biol 1989, 206:397-406.

10. Gianese G, Argos P, Pascarella S: Structural adaptation of enzymes to low temperatures. Protein Eng 2001, 14:141-148.

11. McDonald JH: Patterns of temperature adaptation in proteins from the bacteria Deinococcus radiodurans and Thermus thermophilus. Mol Biol Evol 2001, 18:741-749.

12. Mandrich L, Pezzullo M, Del Vecchio P, Barone G, Rossi M, Manco G Analysis of thermal adaptation in the HSL enzyme family. J Mol Biol 2004, 335:357-369.

13. Metpally RP, Reddy BV: Comparative proteome analysis of psychrophilic versus mesophilic bacterial species: Insights into the molecular basis of cold adaptation of proteins. BMC Genomics 2009, 10:11.

14. Zeldovich KB, Berezovsky IN, Shakhnovich El: Protein and DNA sequence determinants of thermophilic adaptation. PLOS Comput Biol 2007, 3:e5.

15. Berezovsky IN, Zeldovich KB, Shakhnovich El: Positive and negative design in stability and thermal adaptation of natural proteins. Plos Computational Biology 2007, 3:498-507.

16. Gromiha MM, Oobatake M, Sarai A: Important amino acid properties for enhanced thermostability from mesophilic to thermophilic proteins. Biophysical Chemistry 1999, 82:51-67.

17. Mcfallngai MJ, Horwitz J: A Comparative-Study of the Thermal-Stability of the Vertebrate Eye Lens - Antarctic Ice Fish to the Desert Iguana. Experimental Eye Research 1990, 50:703-709.

18. Greaves RB, Warwicker J: Mechanisms for stabilisation and the maintenance of solubility in proteins from thermophiles. Bmc Struct Biol 2007, 7:18

19. Wu LC, Lee JX, Huang HD, Liu BJ, Horng JT: An expert system to predict protein thermostability using decision tree. Expert Syst App/ 2009, 36:9007-9014.

20. Montanucci L, Fariselli P, Martelli PL, Casadio R: Predicting protein thermostability changes from sequence upon multiple mutations. Bioinformatics (Oxford, England) 2008, 24:1190-1195.

21. Gromiha MM, Suresh MX: Discrimination of mesophilic and thermophilic proteins using machine learning algorithms. Proteins-Structure Function and Bioinformatics 2008, 70:1274-1279.

22. Das S, Paul S, Bag SK, Dutta C: Analysis of Nanoarchaeum equitans genome and proteome composition: indications for hyperthermophilic and parasitic adaptation. Bmc Genomics 2006, 7:186.

23. Haney PJ, Badger JH, Buldak GL, Reich Cl, Woese CR, Olsen GJ: Thermal adaptation analyzed by comparison of protein sequences from mesophilic and extremely thermophilic Methanococcus species. P Natl Acad Sci USA 1999, 96:3578-3583.

24. Sadeghi M, Naderi-Manesh $H$, Zarrabi M, Ranjbar B: Effective factors in thermostability of thermophilic proteins. Biophysical Chemistry 2006, $119: 256-270$ 
25. Cambillau C, Claverie JM: Structural and genomic correlates of hyperthermostability. J Biol Chem 2000, 275:32383-32386.

26. Xiao L, Honig B: Electrostatic contributions to the stability of hyperthermophilic proteins. Journal of Molecular Biology 1999, 289:1435-1444.

27. George RA, Heringa J: An analysis of protein domain linkers: their classification and role in protein folding. Protein Eng 2002, 15:871-879.

28. Vogt G, Woell S, Argos P: Protein thermal stability, hydrogen bonds, and ion pairs. J Mol Biol 1997, 269:631-643.

29. Thompson MJ, Eisenberg D: Transproteomic evidence of a loop-deletion mechanism for enhancing protein thermostability. J Mol Biol 1999, 290:595-604.

30. Szilagyi A, Zavodszky P: Structural differences between mesophilic, moderately thermophilic and extremely thermophilic protein subunits: results of a comprehensive survey. Structure 2000, 8:493-504.

31. Maugini E, Tronelli D, Bossa F, Pascarella S: Structural adaptation of the subunit interface of oligomeric thermophilic and hyperthermophilic enzymes. Computational biology and chemistry 2009, 33:137-148.

32. Berezovsky IN, Shakhnovich El: Physics and evolution of thermophilic adaptation. Proc Natl Acad Sci USA 2005, 102:12742-12747.

33. Heaton AL, Ye SJ, Armentrout PB: Experimental and theoretical studies of sodium cation complexes of the deamidation and dehydration products of asparagine, glutamine, aspartic acid, and glutamic acid. The journal of physical chemistry 2008, 112:3328-3338.

34. Xie M, Shahrokh Z, Kadkhodayan M, Henzel WJ, Powell MF, Borchardt RT, Schowen RL: Asparagine deamidation in recombinant human lymphotoxin: hindrance by three-dimensional structures. Journal of pharmaceutical sciences 2003, 92:869-880.

35. Altschul SF, Madden TL, Schaffer AA, Zhang J, Zhang Z, Miller W, Lipman DJ: Gapped BLAST and PSI-BLAST: a new generation of protein database search programs. Nucleic Acids Res 1997, 25:3389-3402.

36. Trivedi S, Gehlot HS, Rao SR: Protein thermostability in Archaea and Eubacteria. Genetics and Molecular Research 2006, 5:816-827.

37. Glyakina AV, Garbuzynskiy SO, Lobanov MY, Galzitskaya OV: Different packing of external residues can explain differences in the thermostability of proteins from thermophilic and mesophilic organisms. Bioinformatics 2007, 23:2231-2238.

38. Haney PJ, Stees M, Konisky J: Analysis of thermal stabilizing interactions in mesophilic and thermophilic adenylate kinases from the genus Methanococcus. J Biol Chem 1999, 274:28453-28458.

39. Jones DT: Protein secondary structure prediction based on positionspecific scoring matrices. Journal of molecular biology 1999, 292:195-202.

40. Cheng J, Randall AZ, Sweredoski MJ, Baldi P: SCRATCH: a protein structure and structural feature prediction server. Nucleic Acids Res 2005, 33:W72-76.

41. Breiman L: Random forests. Machine Learning 2001, 45:5-32.

42. Jain P, Garibaldi JM, Hirst JD: Supervised machine learning algorithms for protein structure classification. Comput Biol Chem 2009, 33:216-223.

43. Han $P$, Zhang $X$, Feng ZP: Predicting disordered regions in proteins using the profiles of amino acid indices. Bmc Bioinformatics 2009, 10(Suppl 1): S42.

44. Breiman L, Friedman J, Olshen R, Stone C: Classification and Regression Trees. Norwell: Kluwer Academic Publishers 1984.

45. Zhang GY, Fang BS: Discrimination of thermophilic and mesophilic proteins via pattern recognition methods. Process Biochemistry 2006, 41:552-556.

46. Fisher RA: On the interpretation of $\chi 2$ from contingency tables, and the calculation of P. Journal of the Royal Statistical Society 1922, 85:87-94.

47. Dubchak I, Holbrook SR, Kim SH: Prediction of Protein Folding Class from Amino-Acid-Composition. Proteins 1993, 16:79-91.

48. Kim H, Moon EJ, Moon S, Jung HJ, Yang YL, Park YH, Heo M, Cheon M, Chang I, Han DS: New method of evaluating relative thermal stabilities of proteins based on their amino acid sequences; Targetstar. International Journal of Modern Physics C 2007, 18:1513-1526.

49. Goihberg E, Dym O, Tel-Or S, Levin I, Peretz M, Burstein Y: A single proline substitution is critical for the thermostabilization of Clostridium beijerinckii alcohol dehydrogenase. Proteins 2007, 66:196-204.

50. Frank Eisenhaber PA: Improved strategy in analytic surface calculation for molecular systems: Handling of singularities and computational efficiency. Journal of Computational Chemistry 1993, 14:1272-1280.

51. Gasteiger E, HC GA, Duvaud S, Wilkins MR, Appel RD, Bairoch A: Protein Identification and Analysis Tools on the ExPASy Server. Book Protein
Identification and Analysis Tools on the ExPASy Server City: Humana Press 2005, 571-607, (Editor ed.^eds).

52. McGuffin $L$ J, Bryson K, Jones DT: The PSIPRED protein structure prediction server. Bioinformatics 2000, 16:404-405.

53. Linding R, Jensen LJ, Diella F, Bork P, Gibson TJ, Russell RB: Protein disorder prediction: implications for structural proteomics. Structure 2003, 11:1453-1459.

doi:10.1186/1471-2105-11-62

Cite this article as: Li et al:: A novel scoring function for discriminating hyperthermophilic and mesophilic proteins with application to predicting relative thermostability of protein mutants. $B M C$

Bioinformatics 2010 11:62.

\section{Submit your next manuscript to BioMed Central and take full advantage of:}

- Convenient online submission

- Thorough peer review

- No space constraints or color figure charges

- Immediate publication on acceptance

- Inclusion in PubMed, CAS, Scopus and Google Scholar

- Research which is freely available for redistribution

Submit your manuscript at www.biomedcentral com/submit
C) Biomed Central 